\title{
Expresión de Ciclooxigenasa-2 en carcinoma colorrectal, una revisión narrativa
}

\author{
Inés Benedetti-Padrón', Daniel Becerra-Mejía
}

\section{RESUMEN}

El desarrollo del carcinoma colorrectal es un proceso secuencial asociado con la inestabilidad cromosómica y con mutaciones de oncogenes como KRAS, de genes supresores de tumor como p53, o con pérdida del gen APC, causando transformación y proliferación celular descontrolada. La ciclooxigenasa-2 (COX-2) es una enzima inducible, cuya expresión puede ser influenciada por estímulos proinflamatorios y mitógenos como los ocasionados por citoquinas y factores de crecimiento. Esta ha sido propuesta como reguladora de la proliferación celular y se ha planteado que puede jugar un papel importante en el desarrollo del tejido metaplásico y displásico, así como en el desarrollo y progresión de diferentes tipos de tumores, entre ellos el carcinoma colorrectal. Se han utilizado diferentes técnicas para identificar el nivel de COX-2 en neoplasias colorrectales, una de las más utilizadas es la inmunohistoquímica, que ha permitido demostrar mayor expresión de la enzima en el tejido tumoral en comparación con la mucosa colorrectal normal. La mayoría de los estudios publicados han sugerido que la sobreexpresión común de COX-2 en el carcinoma colorrectal podría ser utilizada como biomarcador para esta neoplasia.

\section{PALABRAS CLAVE}

Ciclooxigenasa 2; Inmunohistoquímica; Neoplasia ColorrectaI

1 MD, Patóloga PhD en Ciencias Biomédicas, Grupo de investigación Histopatología. Profesor titular Departamento de Ciencias Básicas, Facultad de Medicina, Universidad de Cartagena, Colombia.

2 Estudiante de pregrado, miembro del Semillero de investigación NEO, Grupo de investigación Histopatología, Facultad de Medicina, Universidad de Cartagena, Colombia. Correspondencia: Inés Benedetti-Padrón; ebenedettip1@unicartagena.edu.co

Recibido: febrero 22 de 2018

Aceptado: julio 24 de 2018

Cómo citar: Benedetti-Padrón I, Becerra-Mejía D. Expresión de Ciclooxigenasa-2 en carcinoma colorrectal, una revisión narrativa. latreia. 2019 Ene-Mar;32(1):52-63. D0I 10.17533/ udea.iatreia.v32n1a06. 


\section{SUMMARY}

\section{Expression of Ciclooxigenase-2 in colorectal carci- noma, a narrative review}

The development of colorectal carcinoma is a sequential process associated to chromosomic instability and to mutations of oncogenes as KRAS, of tumor suppressor gene as $p 53$, or loss of $A P C$, leading to uncontrolled cell proliferation and transformation. Cyclooxygenase 2 (COX-2) is an inducible enzyme, whose expression can be influenced by proinflammatory and mitogenic stimuli such as those caused by cytokines and growth factors, has been proposed as an enzyme regulating cell proliferation, and therefore carcinogenesis, which can play an important role in the development of metaplastic and dysplastic tissues, as well as in the development and progression of cancer as colorectal tumors. Different techniques have been used to identify COX-2 expression in colorectal neoplasms, one of the most widely used is the immunohistochemistry, whose results have generally shown higher staining in tumor tissue compared to normal colorectal mucosa. Most published studies have suggested that the common overexpression of COX-2 in colorectal carcinoma could be used as a biomarker for this neoplasm.

\section{KEYWORDS}

\section{Colorectal Neoplasms; Cyclooxygenase-2; Immunohistochemistry}

\section{INTRODUCCIÓN}

El carcinoma colorrectal es una de las neoplasias maliognas de mayor incidencia y mortalidad a nivel mundial (1). En su desarrollo participan múltiples alteraciones moleculares relacionadas con inestabilidad genómica, epigenómica y mutaciones de oncogenes $y$ genes supresores de tumor. Se ha planteado que la enzima ciclooxigenasa 2 (COX-2) juega un papel importante en el desarrollo de tejido metaplásico y displásico, así como en el desarrollo y proogresión del cáncer $(2,3)$. Se ha descrito su sobreexpresión en adenomas y carcinomas colorrectales, $y$ a pesar de haber sido postulada como posible participante en la patogenia de esta neoplasia (4), existe controversia en cuanto a su papel en este proceso. Teniendo en cuenta el alto impacto de esta neoplasia en la carga del cáncer, el objetivo de esta revisión narrativa es presentar la evidencia científica disponible sobre la expresión de COX-2 en el carcinoma colorrectal.

\section{MÉTODOS}

Se llevó a cabo una revisión narrativa de la literatura recopilando información actual sobre la expresión de COX-2 en el carcinoma colorrectal, incluyendo la epidemiología, los factores de riesgo y la patogénesis de esta neoplasia. Se efectuó una búsqueda bibliográfica de artículos en idioma inglés, desde enero de 2012 hasta febrero de 2018, en la base de datos Pubmed, utilizando las palabras clave de búsqueda: COX2, COX 2, COX-2, Cyclooxygenase 2, Colorectal cancer, Epidemiology, Risk factors y Pathogenesis. Se utilizaron las siguientes combinaciones con conectores boleados para la búsqueda: (COX2 OR COX 2 OR COX-2 OR Cyclooxygenase) AND Cancer colorectal, Cancer colorectal AND Epidemiology, Cancer colorectal AND Risk factors y Cancer colorectal AND Pathogenesis. Se completó la búsqueda con la lectura y rastreo de bibliografía referenciada en los documentos seleccionados, además de información encontrađa en artículos con fechas de publicación anterior, debido a su valor para esta revisión. En la Tabla 1 se presenta un resumen de algunos de los estudios sobre Ciclooxigenasa 2 y de cáncer colorrectal incluidos en esta revisión.

\section{RESULTADOS}

\section{Epidemiología, impacto global y local del carcino- ma colorrectal}

El cáncer colorrectal es el tercer tipo de cáncer más común en hombres y el segundo más común en mujeres en el mundo, con 1,4 millones de casos y 694.000 muertes por esta causa anualmente (1). Se presenta una tasa de incidencia ajustada por edad de 17,2 por 100.000 , para un total de 746.000 casos en hombres y 614.000 casos en mujeres (1). La tasa de mortalidad de cáncer colorrectal ajustada por edad es de 8,2 por 100.000 , siendo la cuarta causa de mortalidad por cáncer en ambos sexos. Se reportan cerca de 694.000 muertes al 
año por cáncer colorrectal, representando el 8,5\% de todas las muertes por cáncer en el mundo (1).

La incidencia del cáncer colorrectal varía en el mundo y está relacionada con los hábitos de vida occidentales (5). Cerca del $60 \%$ de los casos ocurren en regiones desarrolladas (1), siendo una patología propia de las zonas urbanas e industrializadas que han modificado su estilo de vida. Las tasas de incidencia más altas se encuentran en Australia y Nueva Zelanda, las más bajas en África Occidental (1). En Ios Estados Unidos, tanto hombres como mujeres de raza negra presentan las tasas de incidencia más altas, seguidos de la población amerindia, y las más bajas se encuentran en la población blanca, hispana, y asiática (6).

En América del Sur, ocupa el tercer lugar en incidencia entre las patologías oncológicas en ambos sexos, siendo el segundo cáncer con mayor mortalidad tanto en hombres como en mujeres (1).

En Colombia, en el año 2015, el cáncer colorrectal ocupó el quinto lugar entre los tumores invasivos, con 10.208 pacientes afectados, el $89,7 \%$ de ellos mayores de 45 años. Se presentaron 1.967 pacientes con diagnóstico de cáncer colorrectal representando el 6,4\% de los casos nuevos de cáncer. En el país, la prevalencia fue de 21,2 por 100.000 y la incidencia de 41 por 100.000 habitantes. El total de pacientes fallecidos por esta neoplasia fue de 1.436 , con una mortalidad de 3 por 100.000 habitantes (7). La población más afectada por grupo de edad se encuentra entre los 65 y 75 años, sin mayores diferencias entre sexos (1). Hay predominio del problema en la región central del país, con mayor riesgo de muerte en ciudades como Cali, Bogotá y Medellín, o en departamentos como Caldas, Risaralda, Valle del Cauca, Antioquia y Santanderes. Mientras que Cauca, Nariño, La Guajira y el Magdalena medio muestran los riesgos de muerte más bajos por esta enfermedad (8). Colombia sigue un patrón similar al observado en países desarrollados, respecto a que las mayores tasas de incidencia y mortalidad de cáncer colorrectal, se presentan en las zonas de mayor urbanización y mayor desarrollo socioeconómico (8). Aunque la incidencia va en aumento, debido al envejecimiento poblacional $y$ factores asociados como la obesidad y los hábitos alimentarios, en general hay una tendencia a la disminución de la mortalidad, probablemente debido a actividades de promoción, prevención y tratamientos oportunos (9).

\section{Factores de riesgo para carcinoma colorrectal}

Los factores de riesgo asociados al cáncer colorrectal se pueden aogrupar en genéticos, ambientales y patologías orgánicas previas predisponentes. Clínicamente, el carcinoma colorrectal se presenta en dos escenarios: el esporádico que corresponde al 80 a $90 \%$ de los casos, al parecer sigue la secuencia adenomacarcinoma; y los individuos con riesgo definido por presentar factores genéticos predisponentes como los antecedentes familiares, la presencia de enfermedades polipósicas y no polipósicas del colon y la enfermedad inflamatoria intestinal (10).

\section{Factores ambientales}

El mayor riesogo de cáncer colorrectal en las zonas más pobladas y con mayor urbanización e industrialización refleja su relación con los factores ambientales. La obesidad, el sedentarismo y la dieta rica en azúcar, carnes rojas, grasas saturadas, carbohidratos refinados $y$ alimentos procesados, aumentan el riesgo de desarrollar cáncer colorrectal (11-16). Los factores ambientales están asociados con los tumores esporádicos y son responsables de aproximadamente el $83 \%$ de todos los casos de cáncer colorrectal (17).

La influencia de los factores ambientales se evidencia en los cambios en las cifras de incidencia de cáncer colorrectal asociados a la migración, las cuales son más altas en las poblaciones del este asiático que migraron a occidente, en comparación con sus contrapartes que permanecieron en Asia (18). Igualmente, Ios mexicanos nacidos en Ios Estados Unidos de América (USA) tienen más del doble de la mortalidad por cáncer colorrectal que los nacidos en México (19).

\section{Factores genéticos}

Aunque la mayoría de los carcinomas colorrectales ocurren en forma esporádica, alrededor de un tercio están relacionados con factores hereditarios (20). EI síndrome de cáncer hereditario comprende el cáncer colorrectal hereditario no polipósico (síndrome de Lynch), responsable de cerca del $3 \%$ de todos los carcinomas colorrectales $y$, la poliposis adenomatosa familiar causada por mutaciones de línea germinal del gen supresor de tumor APC (Adenomatosis poliposis coli), en la que se desarrollan cientos de pólipos adenomatosos colónicos, de los cuales algunos pueden 
evolucionar a un carcinoma invasivo. Las mutaciones somáticas y deleciones que inactivan ambos alelos del gen APC están presentes en la mayoría de los carcinomas colorrectales esporádicos $(21,22)$.

Se ha demostrado la importancia de la interacción genes-ambiente en la incidencia de carcinoma colorrectal, un ejemplo es el caso de los japoneses nacidos en USA que presentan incidencia 40-60 \% más alta de cáncer colorrectal que la de los blancos americanos. Esto puede ser debido a que su predisposición genética a este tumor es menos probable que se manifieste en las condiciones ambientales de su país de origen, pero al adoptar el estilo de vida americano aumenta la exposición a los agentes que los hacen genéticamente susceptibles al desarrollo de esta neoplasia (18).

\section{Patologías predisponentes}

Los pacientes con colitis ulcerativa y enfermedad de Crohn de larga evolución tienen mayor riesgo de desarrollar cáncer colorrectal, este riesgo aumenta en los casos con colangitis esclerosante primaria (23). Además, son considerados de alto riesgo para la presentación de carcinoma colorrectal los pacientes con síndrome de Lynch, Poliposis Adenomatosa Familiar, síndrome de Turcot, síndrome de Gardner y síndrome de Peutz-Jeghers $(17,24,25)$.

\section{Patogenia del carcinoma colorrectal}

Un aspecto principal en la patogenia del carcinoma colorrectal es la acumulación de daño genético y cambios epigenéticos que transforman el epitelio glandular en una lesión tumoral invasiva. Este modelo de progresión tumoral comprende en una etapa inicial la formación de una neoplasia beniona, seguida por la transformación maliona del epitelio que lleva finalmente a una lesión invasiva $(10,26,27)$. Los estudios genéticos de esta secuencia denominada adenoma-displasiacarcinoma han demostrado que no es la única vía de la carcinogénesis colónica que se fundamenta en la acumulación de alteraciones genéticas que promueven la iniciación y progresión de esta neoplasia (22).

El modelo original de la carcinogénesis colónica propuso que solo los adenomas tubulares y túbulo-vellosos podían progresar a adenocarcinoma, sin embargo, actualmente se conoce que los pólipos aserrados también tienen el potencial de sufrir una transformación maligna (28).
Actualmente se considera que la secuencia pólipocáncer en el colon es un proceso heterogéneo en el que participan vías moleculares que se han clasificado como la vía de la inestabilidad cromosómica y la vía del pólipo aserrado (29). En la primera, el cáncer se desarrolla a partir de adenomas tubulares convencionales en los que dicha inestabilidad genómica $y$ epigenómica genera una tasa de mutaciones suficiente en las células epiteliales para el desarrollo de una lesión invasiva, con acumulación de mutaciones progresivas en genes como APC, KRAS, SMAD4 y p53 (29, 30,31 ), lo que conduce a desregulación de vías de señalización que controlan los procesos de proliferación celular, diferenciación, apoptosis, angiogénesis e invasión (32). Entre las vías de señalización específicamente desreguladas en esta neoplasia se encuentran Ia vía WNT-B-catenina, la del factor de crecimiento transformante $\mathrm{B}$, Ia del receptor de factor de crecimiento epidérmico-MAPK y la de fosfatidilinositol 3 quinasa (PI3K) (33). Los carcinomas que se desarrollan por esta vía tienen estabilidad de los microsatélites y no presentan alteración de la reparación del apareamiento (MMR del inglés: mismatch repair). Esta vía corresponde a cerca del $80 \%$ de los carcinomas colorrectales en los Estados Unidos (29).

En la vía molecular de los pólipos aserrados de la carcinogénesis colorrectal el tumor se desarrolla a partir de la transformación maligna de las lesiones precursoras aserradas: adenoma sésil aserrado/pólipo aserrado. Los pólipos aserrados se relacionan con un fenotipo metilador de islas CpG, y esta vía a su vez con mutaciones de BRAF, acumulación de mutaciones asociadas con inestabilidad de los microsatélites y defectos en la reparación del apareamiento por metilación y silenciamiento del gen MLH1. Los pólipos aserrados se asocian con un fenotipo metilador de islas CpG, y esta vía se asocia con mutaciones de BRAF, acumulación de mutaciones asociadas con inestabilidad de los microsatélites y defectos en la reparación del apareamiento por metilación y silenciamiento del gen MLH1 (29).

La inestabilidad de los microsatélites también se presenta en el cáncer colorrectal ligado al síndrome de Lynch, este se origina de adenomas, pero en estos casos la progresión de la secuencia adenoma-carcinoma ocurre más rápidamente, se caracteriza por alteraciones genéticas debidas a la inactivación de los genes MMR (29). 


\section{Ciclooxigenasas y carcinoma colorrectal}

Las ciclooxigenasas (COX) son enzimas codificadas por el gen PTGS1, catalizan la conversión de ácido araquidónico libre en prostaglandina H2, que es el precursor de otras prostaglandinas $y$ tromboxanos (34-36). Estos compuestos reguladores juegan un papel en diversos procesos biológicos como la proliferación celular, angiogénesis, función inmune e inflamación, los cuales se consideran cruciales en el desarrollo y la progresión de algunas neoplasias (2).

La familia de las ciclooxigenasas humanas consta de tres miembros: COX-1, COX-2 y COX-3. COX-1, se encuentra en la mayoría de los tejidos y juega un papel en la homeostasis de muchos procesos fisiológicos. COX-3 es un producto de empalme alternativo de COX-1 y está involucrado en la regulación del dolor y la fiebre. COX-2 es una enzima inducible, cuya expresión puede ser influenciada por estímulos proinflamatorios y mitógenos como los ocasionados por citoquinas $y$ factores de crecimiento (3).

Se ha planteado que COX-2 juega un papel importante en el desarrollo del tejido metaplásico y displásico, así como en el desarrollo y la progresión de cáncer, debido a su participación en la regulación de la proliferación y transformación celular y de la capacidad de crecimiento e invasión de las células tumorales (2, 3). La sobreexpresión de COX-2 se ha asociado con lesiones epiteliales premalionas y malionas, en particular en el tracto gastrointestinal (37-42), los tumores con altos niveles de COX-2 parecen tener un comportamiento más agresivo y se asocian con supervivencia significativamente reducida (43).

Se ha demostrado que el gen que codifica para COX-2 se expresa en el $40 \%$ de los adenomas de colon y en el $90 \%$ de los adenocarcinomas colorrectales, lo cual ha sido asociado con aumento en la producción de factores de crecimiento en estas neoplasias (4). Sin embargo, otros estudios han concluido que no existe relación entre la expresión de COX-2 y la superficie de los pólipos adenomatosos colorrectales, o la de los adenocarcinomas colorrectales $(44,45)$.

Estudios realizados en modelos animales han demostrado la capacidad de COX-2 para promover la carcinogénesis gastrointestinal en el contexto de la pérdida del gen APC. En estudios genéticos se observó que la deleción de COX-2 en modelos de tumorigénesis intestinal en ratones disminuyó la formación de tumores colónicos y de intestino delgado. Además, se describe una asociación entre niveles elevados de $\beta$-catenina nuclear y expresión de COX-2 en células de cáncer de colon humanas y murinas relacionadas con defectos en el gen $\operatorname{APC}(22,46)$.

Aunque los tumores colorrectales esporádicos con frecuencia sobreexpresan COX-2 (47), hasta la mitad de los tumores con deficiencia de genes encargados de la MMR, no muestran sobreexpresión de COX-2. Esto soporta la participación de una vía epigenética en la progresión de la enfermedad (48).

\section{Antiinflamatorios no esteroideos y carcinoma colorrectaI}

Numerosos estudios han propuesto a los antiinflamatorios no esteroideos (AINES) en la prevención del carcinoma colorrectal. Se considera que el principal mecanismo del efecto quimiopreventivo de estos compuestos es la inhibición de COX-2 (49-51). La evidencia apunta a que inducen regresión de los adenomas, observándose menor frecuencia de estos y de cáncer colorrectal en individuos con ingesta regular de AINES $(4,37)$. Se ha demostrado que el uso de celecoxib y sulindac, reduce la formación de adenomas en pacientes con poliposis colónica familiar y disminuye la formación de adenomas colorrectales en pacientes con cáncer colorrectal esporádico (46). En niños con poliposis colónica familiar el tratamiento con celecoxib durante 5 años se asoció con menor tasa de poliposis y mayor tiempo de progresión de la enfermedad (52).

Algunos autores sugieren que estos medicamentos juegan un papel importante en la infiltración tumoral por células del sistema inmune $(\mathrm{T}$ CD3,$+ \mathrm{CD} 8+y$ CD45+), con menor tasa de infiltración por estas céIulas inmunitarias en muestras de cáncer colorrectal de pacientes que tomaban AINES, comparadas con las muestras de pacientes sin ingesta de estos (49, 53). El uso de AINES también se ha relacionado con menor frecuencia de otras neoplasias epiteliales gastrointestinales, por ejemplo, en un modelo animal se observó menor frecuencia de adenocarcinoma esofágico al suprimir, mediante fármacos, los niveles de COX-2 en animales de laboratorio sometidos a reflujo gastroesofágico, con respecto al grupo control. Se ha descrito también una relación entre su uso y menor 
frecuencia de otras neoplasias epiteliales gastrointestinales, por ejemplo en un modelo animal se observó menor frecuencia de adenocarcinoma esofágico en animales de laboratorio, sometidos a reflujo gastroesofágico al suprimir mediante fármacos los niveles de COX-2 (54).

Los hallazgos de la sobreexpresión de COX-2 en muchas lesiones premalignas, en combinación con los beneficios informados de los efectos de los AINES en la prevención del cáncer, sugieren que su inhibición puede ser crucial en este proceso (34).

Aunque se ha demostrado que los inhibidores de COX disminuyen el riesgo de cáncer colorrectal, su uso prolongado se ha asociado con efectos cardiovasculares adversos (46), por lo que algunos estudios sobre inhibidores de COX y adenomas tuvieron que ser interrumpidos $(55,56)$. Desafortunadamente, los pacientes con mayor riesgo en el tratamiento con inhibidores de COX (con obesidad y vida sedentaria, entre otros) son también los que tienen mayor riesgo de desarrollar carcinoma colorrectal, y los que más se beneficiarían de una prevención primaria que, en su caso, puede suponer mayores efectos secundarios indeseables (55).

\section{Detección de expresión de COX-2 en carcinoma colorrectal}

La detección temprana de cualquier tipo de cáncer mejora la supervivencia de los individuos que lo padecen. Esto cobra mayor importancia en tumores con alta letalidad como el carcinoma colorrectal (57). Se han detectado altos niveles de COX-2 en tumores colorrectales tanto en humanos como en animales, mientras que en la mucosa intestinal normal su expresión es muy baja o indetectable. COX-2 se ha descrito sobreexpresada en aproximadamente el $80 \%$ de los carcinomas colorrectales (22).

La expresión de COX-2 ha sido asociada con la detección temprana y el pronóstico del carcinoma colorrectal. Varios estudios describen que COX-2 se localiza predominantemente en las células estromales en adenomas intestinales humanos y de roedores, y en las células epiteliales displásicas que eventualmente sufren transformación maliogna, demostrando la posible utilidad de su identificación en el tejido tumoral en etapas tempranas del carcinoma colorrectal
(58-62). Los niveles aumentados de COX-2 en el carcinoma colorrectal se han asociado con metástasis hepáticas y mal pronóstico de estos pacientes (63).

Dado que la expresión no regulada de COX-2 puede ocurrir en las fases tempranas de la carcinogénesis colorrectal (22), es crucial estimar su nivel de manera confiable (64). Se han utilizado diferentes técnicas para determinar los niveles de COX-2 en neoplasias colorrectales, como el ensayo cuantitativo de los niveles de ARNm de COX-2, y la tinción de inmunohistoquímica utilizando diferentes anticuerpos para detectar esta enzima $(38,65,66)$.

Existen variaciones en los resultados de los estudios que han realizado análisis de ARNm de COX-2 en carcinoma colorrectal. En la mayoría de ellos, el ARNm de COX-2 se encontró elevado en el tejido tumoral, aunque en un gran porcentaje el número de muestras utilizadas fue pequeño $(39,66-68)$. Mientras que en dos estudios con mayor número de muestras (99 y 46 muestras de tumor, respectivamente) no se encontró aumento de los niveles de ARNm de COX-2 en el tejido tumoral $(65,69)$.

Entre las investigaciones con bajo número de muestras, Eberhart et al., informaron niveles elevados de COX-2 en 3 de 6 adenomas colorrectales en comparación con la mucosa normal (39). Por su parte, Nosho et al., en un mayor número de casos encontraron mayor nivel de ARNm de COX-2 en el $42 \%$ de los tumores colorrectales, entre 63 adenomas y 27 carcinomas (67). Mientras que, Roelofs, et aI. (34), reportan aumento en el ARNm de COX-2 en el $77 \%$ de los carcinomas colorrectales, lo que sugiere que dicha sobreexpresión es menor en los adenomas colorrectales que en los carcinomas y aumenta en algún punto de la transición entre adenoma y carcinoma $(70,71)$.

En cuanto a la detección de expresión de COX-2 mediante inmunohistoquímica, la evaluación de la inmunotinción de COX-2 en los tejidos toma en cuenta dos aspectos: la intensidad y la extensión de la tinción. La intensidad de la tinción es evaluada como 0 (negativa), 1 (débil), 2 (moderada) y 3 (fuerte). La extensión de la tinción se califica como 0 (0 \%), 1 (1-25\%), 2 (26-50\%), 3 (51-75\%) y 4 (76-100 \%) según los porcentajes del área de tinción positiva en relación con el área de carcinoma. La suma del puntaje de intensidad y de extensión resulta en un puntaje de tinción final (0-7), en el que los 
tumores con puntaje mayor a 2 se consideran positivos para COX-2 $(72,73)$.

En varias investigaciones, la evaluación mediante inmunohistoquímica ha mostrado mayor porcentaje de tinción en el tejido tumoral en comparación con la mucosa colorrectal normal, con una variabilidad de la positividad de la tinción en el tumor que va desde 35 a 62 \% en cánceres colorrectales esporádicos a 100 $\%$ en tumores colorrectales de pacientes con poliposis adenomatosa familiar $(65,67,73,74)$. Mientras que, otras han reportado que la sobreexpresión de COX-2 estaba presente solo en 21-25\% de los cánceres colorrectales $(72,75,76)$.

Otros estudios han concluido que no existe relación entre la expresión de COX-2 y la superficie de los pólipos adenomatosos colorrectales $y$ los carcinomas colorrectales. Además, sugieren que no hay asociación significativa del nivel de COX-2 con la supervivencia del paciente y el pronóstico del cáncer colorrectal (44, 45). Estas diferencias pueden relacionarse con la variación del sistema de puntuación y/o los niveles de corte utilizados, como en la evaluación estadística realizada por Lim et al., donde solo los tumores con puntaje de tinción mayor o igual a 3 se consideraron positivos, disminuyendo notablemente la utilidad de la inmunotinción para COX-2 en los tumores examinados (44).

Otra explicación podría ser que los niveles de COX-2 están asociados con mayor tamaño tumoral, invasión más profunda y presencia de metástasis a nódulos linfáticos en el carcinoma colorrectal, por lo que en los de pequeño tamaño o en etapas tempranas, su expresión estaría disminuida $(72,77,78)$.

\section{Tabla 1. Resumen de algunos de los estudios sobre Ciclooxigenasa 2 y de cáncer colorrectal incluidos en esta revisión}

\begin{tabular}{|c|c|c|c|}
\hline Autor (Año) & $\begin{array}{l}\text { Número de } \\
\text { pacientes o } \\
\text { especímenes }\end{array}$ & Conclusiones & Referencia \\
\hline $\begin{array}{l}\text { Hennie MJ } \\
\text { Roelofs et al. } \\
\quad(2014)\end{array}$ & 60 & $\begin{array}{l}\text { Los niveles de ARNm de COX-2 cuantificados por qPCR y normalizados con respecto al } \\
\text { peso del tejido, se encontraron sobre expresados en } 77 \% \text { de los carcinomas colorrectales } \\
\text { en comparación con la mucosa colorrectal sana. Los niveles de COX-2 en el carcinoma se } \\
\text { encontraron casi } 22 \text { veces más altos que los niveles de mucosas normales. }\end{array}$ & 34 \\
\hline $\begin{array}{l}\text { Hsi-Hsien Hsu } \\
\text { et al. (2017) }\end{array}$ & $\begin{array}{l}\text { Cultivo de } \\
\text { células LoVo }\end{array}$ & $\begin{array}{l}\text { EP2 y EP4 median la expresión de COX-2 inducida por PGE2, y la migración celular en } \\
\text { células LoVo de cáncer de colon correlacionadas con la el nivel de COX-2. } \beta \text {-catenina inte- } \\
\text { ractúa en el promotor COX-2 para activar la transcripción de COX-2. }\end{array}$ & 35 \\
\hline $\begin{array}{l}\text { Xiao-Hang Che } \\
\text { et al. (2016) }\end{array}$ & 94 & $\begin{array}{l}\text { Los resultados sugieren que el uso del doble inhibidor de la COX-2/5-LOX, darbufelone, } \\
\text { puede ser un método eficaz para el tratamiento del cáncer de colon. }\end{array}$ & 36 \\
\hline $\begin{array}{l}\text { Sung-Chul Lim } \\
\text { et al. (2008) }\end{array}$ & 231 & $\begin{array}{l}\text { Los hallazgos sugieren que la expresión de COX-2 no desempeña un papel en el pronósti- } \\
\text { co del cáncer colorrectal y no está afectada por el estado de acumulación de p53 nuclear } \\
\text { en esta neoplasia. }\end{array}$ & 44 \\
\hline $\begin{array}{l}\text { Deniz Yamac et } \\
\quad \text { al. (2005) }\end{array}$ & 83 & $\begin{array}{l}\text { Los autores del estudio concluyen que el aumento de los niveles de COX-2 no se correla- } \\
\text { ciona con las características clínico-patológicas del carcinoma de colon ni con el pronósti- } \\
\text { co de esta enfermedad. }\end{array}$ & 45 \\
\hline $\begin{array}{l}\text { Masahiro } \\
\text { Yoshinaga et al. } \\
\text { (2010) }\end{array}$ & 95 & $\begin{array}{c}\text { La expresión de PPAR-delta y COX-2 ha sido asociada con CXCR4 y/o VEGF-A, que están } \\
\text { asociadas con metástasis hepáticas y mal pronóstico en pacientes con cáncer colorrec- } \\
\text { tal. Ambas pueden jugar un papel predictor en el pronóstico de pacientes con cáncer } \\
\text { colorrectal. }\end{array}$ & 68 \\
\hline $\begin{array}{l}\text { Ts. Loukanov et } \\
\text { al. (2010) }\end{array}$ & 26 & $\begin{array}{c}\text { COX-2 podría estar involucrada en la promoción tumoral durante la progresión del cáncer } \\
\text { colorrectal. }\end{array}$ & 67 \\
\hline $\begin{array}{l}\text { Y. Hamaya et al. } \\
\qquad(2010)\end{array}$ & 78 & $\begin{array}{l}\text { La expresión de ARNm de COX-2 en heces de pacientes con cáncer colorrectal, se originó } \\
\text { a partir del tumor o de la mucosa en torno a este. La cantidad de ARNm de COX-2 en he- } \\
\text { ces, fue afectada por múltiples factores, incluido un mayor número de células exfoliadas } \\
\text { del tumor, exfoliación de células inflamatorias, el tamaño tumoral y el nivel de ARNm de } \\
\text { COX-2 en el tejido tumoral, sin embargo, no por la ubicación del tumor. }\end{array}$ & 71 \\
\hline
\end{tabular}




\section{CONCLUSIÓN}

Aunque algunos autores no han encontrado relación entre la expresión de COX-2 y la superficie de los pólipos adenomatosos y los carcinomas colorrectales, y niegan una asociación significativa entre su nivel y el pronóstico de esta neoplasia, numerosas evidencias soportan el papel probable de esta enzima en la carcinogénesis colorrectal. La mayoría de los estudios han demostrado su sobreexpresión y los inhibidores de COX-2 están entre los agentes más efectivos en su prevención. La información publicada sugiere que COX2 podría ser utilizada como un biomarcador de riesgo en el carcinoma colorrectal, futuros estudios respecto a este tema deberán enfocarse a inhibidores de COX2 sin los efectos secundarios de los existentes, como una posible opción preventiva del cáncer colorrectal.

\section{CONFLICTOS DE INTERESES}

Ninguno por declarar.

\section{REFERENCIAS BIBLIOGRÁFICAS}

1. Ferlay J, Soerjomataram I, Dikshit R, Eser S, Mathers $C$, Rebelo $M$, et al. Cancer incidence and mortality worldwide: sources, methods and major patterns in GLOBOCAN 2012. Int J Cancer. 2015 Mar;136(5):E359-86. DOI 10.1002/ijc.29210.

2. Chandrasekharan NV, Simmons DL. The cyclooxygenases. Genome Biol. 2004;5(9):241. DOI 10.1186/gb2004-5-9-241.

3. Chandrasekharan NV, Dai H, Roos KL, Evanson NK, Tomsik J, Elton TS, et al. COX-3, a cyclooxygenase-1 variant inhibited by acetaminophen and other analgesic/antipyretic drugs: cloning, structure, and expression. Proc Natl Acad Sci U S A. 2002 Oct 15;99(21):13926-31. DOI 10.1073/pnas.162468699.

4. Baghaei R, Beiraghdar M, Sobhani A, Rafei R, Kolahi L, Foladi L. An investigation of the rate of cyclooxygenase-2 expression on the surface of adenomatous and colorectal adenocarcinoma polyps. Adv Biomed Res. 2015 Sep;4:200. DOI 10.4103/2277-9175.166136.

5. Brenner H, Kloor M, Pox CP. Colorectal cancer. Lancet. 2014 Apr;383(9927):1490-502. DOI 10.1016/S01406736(13)61649-9.
6. Siegel RL, Miller KD, Jemal A. Cancer Statistics, 2017. CA Cancer J Clin. 2017 Jan;67(1):7-30. DOI 10.3322/ caac.21387.

7. Fondo Colombiano de Enfermedades de Alto Costo Cuenta de Alto Costo. Situación del cáncer en la población adulta atendida en el SGSSS de Colombia 2016 [Internet]. Bogotá: 2017. Disponible en: https://cuentadealtocosto.org/site/images/Libro\%20 C\%C3\%A1ncer\%202015/Situacion_Cancer_Poblacion_Adulta_Web\%20actualizacion_2016.pdf

8. Pardo C, de Vries E, Buitrago L, Gamboa O. Atlas de Mortalidad por cáncer en Colombia [Internet]. $4^{\mathrm{a}}$ ed. Bogotá: Instituto Nacional de Cancerología; 2017. Disponible en: http://www.cancer.gov.co/ATLAS_de_ Mortalidad_por_cancer_en_Colombia.pdf

9. Ministerio de Salud y Protección Social, Colciencias, Instituto Nacional de Cancerología ESE-FEDESALUD. Guía de práctica clínica (GPC) para la detección temprana, diagnóstico, tratamiento, seguimiento y rehabilitación de pacientes con diagnóstico de cáncer de colon y recto [Internet]. Bogotá: Minsalud; 2013. Disponible en: https://www.minsalud.gov.co/sites/rid/ Lists/BibliotecaDigital/RIDE/INEC/IETS/GPC_Comple_Ca_Colon\%20(1).pdf

10. Vogelstein B, Fearon ER, Hamilton SR, Kern SE, Preisinger AC, Leppert $M$, et al. Genetic alterations during colorectal-tumor development. N Engl J Med. 1988 Sep;319(9):525-32. DOI 10.1056/ NEJM198809013190901.

11. Center MM, Jemal A, Smith RA, Ward E. Worldwide variations in colorectal cancer. CA Cancer J Clin. 2009 Nov-Dec;59(6):366-78. DOI 10.3322/caac.20038.

12. World Cancer Research Fund, American Institute for Cancer Research. Food, Nutrition, Physical Activity, and the Prevention of Cancer: a Global Perspective [Internet]. Washington: Cancer Research; 2007. Available from: http://www.aicr.org/assets/docs/pdf/ reports/Second_Expert_Report.pdf

13. Gunter MJ, Leitzmann MF. Obesity and colorectal cancer: epidemiology, mechanisms and candidate genes. J Nutr Biochem. 2006 Mar;17(3):145-56. DOI 10.1016/j.jnutbio.2005.06.011.

14. Moghaddam AA, Woodward M, Huxley R. Obesity and risk of colorectal cancer: a meta-analysis of 31 studies with 70,000 events. Cancer Epidemiol Biomarkers Prev. 2007 Dec;16(12):2533-47. 
15. Azeem S, Gillani SW, Siddiqui A, Jandrajupalli SB, Poh V, Syed Sulaiman SA. Diet and Colorectal Cancer Risk in Asia--a Systematic Review. Asian Pac J Cancer Prev. 2015;16(13):5389-96.

16. Arnold M, Sierra MS, Laversanne M, Soerjomataram I, Jemal A, Bray F. Global patterns and trends in colorectal cancer incidence and mortality. Gut. 2017 Apr;66(4):683-91. DOI 10.1136/gutjnI-2015-310912.

17. Witold K, Anna K, Maciej T, Jakub J. Adenomas - Genetic factors in colorectal cancer prevention. Rep Pract Oncol Radiother. 2018 Mar-Apr;23(2):75-83. DOI 10.1016/j.rpor.2017.12.003.

18. Flood DM, Weiss NS, Cook LS, Emerson JC, Schwartz SM, Potter JD. Colorectal cancer incidence in Asian migrants to the United States and their descendants. Cancer Causes Control. 2000 May;11(5):403-11.

19. Pinheiro PS, Callahan KE, Stern MC, de Vries E. Miorration from Mexico to the United States: A hiog-speed cancer transition. Int J Cancer. 2018 Feb;142(3):47788. DOI 10.1002/ijc.31068.

20. Lichtenstein P, Holm NV, Verkasalo PK, Iliadou A, Kaprio J, Koskenvuo M, et al. Environmental and heritable factors in the causation of cancer--analyses of cohorts of twins from Sweden, Denmark, and Finland. N Engl J Med. 2000 Jul;343(2):78-85.

21. Grover S, Kastrinos F, Steyerberg EW, Cook EF, Dewanwala A, Burbidge LA, et al. Prevalence and phenotypes of APC and MUTYH mutations in patients with multiple colorectal adenomas. JAMA. 2012 Aug;308(5):485-92. DOI 10.1001/jama.2012.8780.

22. Dixon DA, Blanco FF, Bruno A, Patrionani P. Mechanistic aspects of COX-2 expression in colorectal neoplasia. Recent Results Cancer Res. 2013;191:7-37. DOI 10.1007/978-3-642-30331-9_2.

23. Clarke WT, Feuerstein JD. Updates in colorectal cancer screening in inflammatory bowel disease. Curr Opin Gastroenterol. 2018 Jul;34(4):208-16. DOI 10.1097/MOG.0000000000000448.

24. Waller A, Findeis S, Lee MJ. Familial Adenomatous Polyposis. J Pediatr Genet. 2016 Jun;5(2):78-83. DOI 10.1055/s-0036-1579760.

25. Lorans M, Dow E, Macrae FA, Winship IM, Buchanan DD. Update on Hereditary Colorectal Cancer: Improving the Clinical Utility of Multigene Panel Testing. Clin Colorectal Cancer. 2018 Jun;17(2):e293-e305. DOI 10.1016/j.clcc.2018.01.001.
26. Fearon ER, Vogelstein B. A genetic model for colorectal tumorigenesis. Cell. 1990 Jun;61(5):759-67. DOI 10.1016/0092-8674(90)90186-I.

27. Leslie A, Carey FA, Pratt NR, Steele RJ. The colorectal adenoma-carcinoma sequence. $\mathrm{Br} \mathrm{J}$ Surg. 2002 Jul;89(7):845-60. DOI 10.1046/j.13652168.2002.02120.x.

28. Bettington M, Walker N, Clouston A, Brown I, Leggett $B$, Whitehall $\nabla$. The serrated pathway to colorectal carcinoma: current concepts and challenges. Histopathology. 2013 Feb;62(3):367-86. DOI 10.1111/his.12055.

29. Gibson JA, Odze RD. Pathology of premalignant coIorectal neoplasia. Dig Endosc. 2016 Apr;28(3):312-23. DOI 10.1111/den.12633.

30. Burnett-Hartman AN, Newcomb PA, Potter JD, Passarelli MN, Phipps AI, Wurscher MA, et al. Genomic aberrations occurring in subsets of serrated colorectal lesions but not conventional adenomas. Cancer Res. 2013 May;73(9):2863-72. DOI 10.1158/0008-5472. CAN-12-3462.

31. Pino MS, Chung DC. The chromosomal instability pathway in colon cancer. Gastroenterology. 2010 Jun;138(6):2059-72. DOI 10.1053/j.gastro.2009.12.065.

32. Fouad YA, Aanei C. Revisiting the hallmarks of cancer. Am J Cancer Res. 2017 May;7(5):1016-1036.

33. Grady WM, Markowitz SD. The molecular pathogenesis of colorectal cancer and its potential application to colorectal cancer screening. Dig Dis Sci. 2015 Mar;60(3):762-72. DOI 10.1007/s10620-014-3444-4.

34. Roelofs HM, Te Morsche RH, van Heumen BW, Nagengast FM, Peters WH. Over-expression of COX-2 mRNA in colorectal cancer. BMC Gastroenterol. 2014 Jan;14:1. DOI 10.1186/1471-230X-14-1.

35. Hsu HH, Lin YM, Shen CY, Shibu MA, Li SY, Chang SH, et al. Prostaglandin E2-Induced COX-2 Expressions via EP2 and EP4 Signaling Pathways in Human LoVo Colon Cancer Cells. Int J Mol Sci. 2017 May; 18(6). pii: E1132. DOI 10.3390/ijms18061132.

36. Che XH, Chen CL, Ye XL, Weng GB, Guo XZ, Yu WY, et al. Dual inhibition of COX-2/5-LOX blocks colon cancer proliferation, migration and invasion in $\nabla \mathrm{i}-$ tro. Oncol Rep. 2016 Mar;35(3):1680-8. DOI 10.3892/ or.2015.4506.

37. Fujimura T, Ohta T, Oyama K, Miyashita T, Miwa K. Role of cyclooxygenase-2 in the carcinogenesis of 
gastrointestinal tract cancers: a review and report of personal experience. World J Gastroenterol. 2006 Mar;12(9):1336-45. DOI 10.3748/wjo. $\nabla 12.19 .1336$.

38. Sano H, Kawahito Y, Wilder RL, Hashiramoto A, Mukai S, Asai K, et al. Expression of cyclooxygenase-1 and -2 in human colorectal cancer. Cancer Res. 1995 Sep;55(17):3785-9.

39. Eberhart CE, Coffey RJ, Radhika A, Giardiello FM, Ferrenbach S, DuBois RN. Up-regulation of cyclooxygenase 2 gene expression in human colorectal adenomas and adenocarcinomas. Gastroenterology. 1994 Oct; 107(4):1183-8. DOI S0016508594003033.

40. Brown JR, DuBois RN. COX-2: a molecular target for colorectal cancer prevention. J Clin Oncol. 2005 Apr;23(12):2840-55. DOI 10.1200/JCO.2005.09.051.

41. Mehta S, Boddy A, Johnson IT, Rhodes M. Systematic review: Cyclo-oxygenase-2 in human oesophageal adenocarcinogenesis. Aliment Pharmacol Ther. 2006 Nov;24(9):1321-31. DOI 10.1111/j.13652036.2006.03119.x.

42. Liu X, Li P, Zhang ST, You H, Jia JD, Zu ZL. COX-2 mRNA expression in esophageal squamous cell carcinoma (ESCC) and effect by NSAID. Dis Esophagus. 2008;21(1):9-14. DOI 10.1111/j.1442-2050.2007.00697.x.

43. Buskens CJ, Van Rees BP, Sivula A, Reitsma JB, Haglund C, Bosma PJ, et al. Prognostic significance of elevated cyclooxygenase 2 expression in patients with adenocarcinoma of the esophagus. Gastroenterology. 2002 Jun;122(7):1800-7. DOI 10.1053/gast.2002.33580.

44. Lim SC, Lee TB, Choi CH, Ryu SY, Min YD, Kim KJ. Prognostic siognificance of cyclooxygenase-2 expression and nuclear p53 accumulation in patients with colorectal cancer. J Surg Oncol. 2008;97(1):51-56, DOI: $10.1002 /$ jso.20907.

45. Yamac D, Celenkoglu G, Coskun U, Akyurek N, Akcali Z, Dursun A, et al. Prognostic importance of COX-2 expression in patients with colorectal cancer. Pathol Res Pract. 2005;201(7):497-502. DOI 10.1016/j. prp.2005.04.006.

46. Wang Y, Wang W, Sanidad KZ, Shih PA, Zhao X, Zhang G. Eicosanoid signaling in carcinogenesis of colorectal cancer. Cancer Metastasis Rev. 2018 Sep;37(23):257-267. DOI 10.1007/s10555-018-9739-8.

47. Wang D, Dubois RN. The role of COX-2 in intestinal inflammation and colorectal cancer. Oncogene. 2010 Feb 11;29(6):781-8. DOI 10.1038/onc.2009.421.
48. Castells A, Payá A, Alenda C, Rodríguez-Moranta F, Agrelo R, Andreu M, et al. Cyclooxygenase 2 expression in colorectal cancer with DNA mismatch repair deficiency. Clin Cancer Res. 2006 Mar; 12(6):1686-92.

49. Grancher A, Michel P, Di Fiore F, Sefrioui D. [Aspirin and colorectal cancer]. Bull Cancer. 2018 Feb;105(2):171180. DOI 10.1016/j.bulcan.2017.09.013. French.

50. Boutaud O, Sosa IR, Amin T, Oram D, AdIer D, Hwang HS, et al. Inhibition of the Biosynthesis of Prostaglandin E2 By Low-Dose Aspirin: Implications for Adenocarcinoma Metastasis. Cancer Prev Res (Phila). 2016 Nov;9(11):85565. DOI 10.1158/1940-6207.CAPR-16-0094.

51. Zumwalt TJ, Wodarz D, Komarova NL, Toden S, Turner J, Cardenas J, et al. Aspirin-Induced Chemoprevention and Response Kinetics Are Enhanced by PIK3CA Mutations in Colorectal Cancer Cells. Cancer Prev Res (Phila). 2017 Mar;10(3):208-18. DOI 10.1158/19406207.CAPR-16-0175.

52. Burke CA, Phillips R, Berger MF, Li C, Essex MN, Iorga $\mathrm{D}$, et al. Children's International Polyposis (CHIP) study: a randomized, double-blind, placebo-controlled study of celecoxib in children with familial adenomatous polyposis. Clin Exp Gastroenterol. 2017 JuI;10:177-85. DOI 10.2147/CEG.S121841.

53. Cao Y, Nishihara R, Qian ZR, Song M, Mima K, Inamura K, et al. Regular Aspirin Use Associates With Lower Risk of Colorectal Cancers With Low Numbers of Tumor-Infiltrating Lymphocytes. Gastroenterology. 2016 Nov;151(5):879-92.e4. DOI 10.1053/j.gastro.2016.07.030.

54. Buttar NS, Wang KK, Leontovich O, Westcott JY, Pacifico RJ, Anderson MA, et al. Chemoprevention of esophageal adenocarcinoma by COX-2 inhibitors in an animal model of Barrett's esophagus. Gastroenterology. 2002 Apr;122(4):1101-12. DOI 10.1053/ gast.2002.32371.

55. Fernández-Calderón M, Betés Ibáñez MT. La aspirina en la prevención primaria del cáncer colorectal. An Sist Sanit Navar. 2012;35(2):261-7. DOI 10.4321/S113766272012000200008.

56. Bertagnolli MM, Eagle CJ, Zauber AG, Redston M, Solomon SD, Kim K, et al. Celecoxib for the prevention of sporadic colorectal adenomas. N Engl J Med. 2006 Aug;355(9):873-84. DOI 10.1056/NEJMoa061355.

57. Allemani C, Weir HK, Carreira H, Harewood R, Spika D, Wang XS, et al. Global surveillance of cancer survival 1995-2009: analysis of individual data for $25,676,887$ 
patients from 279 population-based registries in 67 countries (CONCORD-2). Lancet. 2015 Mar;385(9972):9771010. DOI 10.1016/S0140-6736(14)62038-9.

58. Hull MA, Booth JK, Tisbury A, Scott N, Bonifer C, Markham AF, et al. Cyclooxygenase 2 is up-regulated and localized to macrophages in the intestine of Min mice. Br J Cancer. 1999 Mar;79(9-10):1399-405.

59. Bamba H, Ota S, Kato A, Adachi A, Itoyama S, Matsuzaki F. High expression of cyclooxygenase-2 in macrophages of human colonic adenoma. Int J Cancer. 1999 Nov;83(4):470-5.

60. Chapple KS, Cartwright EJ, Hawcroft G, Tisbury A, Bonifer C, Scott N, et al. Localization of cyclooxygenase-2 in human sporadic colorectal adenomas. Am J Pathol. 2000 Feb;156(2):545-53. DOI 10.1016/S00029440(10)64759-1.

61. Sonoshita M, Takaku K, Oshima M, Sugihara K, Taketo MM. Cyclooxygenase-2 expression in fibroblasts and endothelial cells of intestinal polyps. Cancer Res. 2002 Dec 1;62(23):6846-9.

62. Adegboyega PA, Ololade O, Saada J, Mifflin R, Di Mari JF, Powell DW. Subepithelial myofibroblasts express cyclooxygenase-2 in colorectal tubular adenomas. Clin Cancer Res. 2004 Sep;10(17):5870-9. DOI 10.1158/1078-0432.CCR-0431-03.

63. Yoshinaga M, Taki K, Somada S, Sakiyama Y, Kubo $\mathrm{N}$, Kaku T, et al. The expression of both peroxisome proliferator-activated receptor delta and cyclooxygenase-2 in tissues is associated with poor prognosis in colorectal cancer patients. Dig Dis Sci. 2011 Apr;56(4):1194-200. DOI 10.1007/s10620-010-1389-9.

64. Greenhough A, Smartt HJ, Moore AE, Roberts HR, WiIliams AC, Paraskeva C, et al. The COX-2/PGE2 pathway: key roles in the hallmarks of cancer and adaptation to the tumour microenvironment. Carcinogenesis. 2009 Mar;30(3):377-86. DOI 10.1093/carcin/bgpo14.

65. Antonacopoulou AG, Tsamandas AC, Petsas T, Liava A, Scopa CD, Papavassiliou AG, et al. EGFR, HER-2 and COX-2 levels in colorectal cancer. Histopathology. 2008 Dec;53(6):698-706.

66. Loukanov Ts, Kirilov M, Fürstenberger G, Müller-Decker K. Localization of cyclo-oxygenase-2 in human recurrent colorectal cancer. Clin Invest Med. 2010 Feb;33(1):E22-9.

67. Nosho K, Yoshida M, Zamamoto H, Taniguchi H, Adachi Y, Mikami M, et al. Association of Ets-related transcriptional factor E1AF expression with overexpression of matrix metalloproteinases, COX-2 and iNOS in the early stage of colorectal carcinogenesis. Carcinogenesis. 2005 May;26(5):892-9. DOI 10.1093/ carcin/bgio29.

68. Delage B, Rullier A, Capdepont M, Rullier E, Cassand P. The effect of body weight on altered expression of nuclear receptors and cyclooxygenase-2 in human colorectal cancers. Nutr J. 2007 Sep;6:20. DOI 10.1186/1475-2891-6-20.

69. Gustafsson A, Hansson E, Kressner U, Nordgren S, Andersson M, Wang W, et al. EP1-4 subtype, COX and PPAR gamma receptor expression in colorectal cancer in prediction of disease-specific mortality. Int J Cancer. 2007 Jul 15;121(2):232-40. DOI 10.1002/ ijc. 22582 .

70. Hamaya X, Yoshida K, Takai T, Ikuma M, Hishida A, Kanaoka S. Factors that contribute to faecal cyclooxygenase-2 mRNA expression in subjects with colorectal cancer. Br J Cancer. 2010 Mar;102(5):916-21. DOI 10.1038/sj.bjc.6605564.

71. Kanaoka S, Yoshida K, Miura N, Sugimura H, Kajimura $M$. Potential usefulness of detecting cyclooxygenase 2 messenger RNA in feces for colorectal cancer screening. Gastroenterology. 2004 Aug;127(2):422-7. DOI 10.1053/j.gastro.2004.05.022.

72. Masunaga R, Kohno H, Dhar DK, Ohno S, Shibakita $\mathrm{M}$, Kinugasa $\mathrm{S}$, et al. Cyclooxygenase-2 expression correlates with tumor neovascularization and prognosis in human colorectal carcinoma patients. Clin Cancer Res. 2000 Oct;6(10):4064-8.

73. Zimmermann KC, Sarbia M, Weber AA, Borchard F, Gabbert HE, Schrör K. Cyclooxygenase-2 expression in human esophageal carcinoma. Cancer Res. 1999 Jan;59(1):198-204.

74. Brosens LA, Keller JJ, Pohjola L, Haglund C, Morsink $\mathrm{FH}$, Iacobuzio-Donahue $\mathrm{C}$, et al. Increased expression of cytoplasmic HuR in familial adenomatous polyposis. Cancer Biol Ther. 2008 Mar;7(3):424-7.

75. Konno H, Baba M, Shoji T, Ohta M, Suzuki S, Nakamura S. Cyclooxygenase-2 expression correlates with uPAR levels and is responsible for poor prognosis of colorectal cancer. Clin Exp Metastasis. 2002;19(6):527-34.

76. Tomozawa S, Tsuno NH, Sunami E, Hatano K, Kitayama J, Osada T, et al. Cyclooxygenase-2 overexpression correlates with tumour recurrence, 
especially haematogenous metastasis, of colorectal cancer. Br J Cancer. 2000 Aug;83(3):324-8. DOI 10.1054/bjoc.2000.1270.

77. Soumaoro LT, Uletake H, Higuchi T, Takagi Y, Enomoto M, Sugihara K. Cyclooxygenase-2 expression: a significant prognostic indicator for patients with colorectal cancer. Clin Cancer Res. 2004 Dec;10(24):8465-71. DOI 10.1158/1078-0432.CCR-04-0653.

78. Fujita T, Matsui M, Takaku K, Uetake H, Ichikawa W, Taketo MM, et al. Size- and invasion-dependent increase in cyclooxygenase 2 levels in human colorectal carcinomas. Cancer Res. 1998 Nov;58(21):4823-6. 